$\begin{array}{ll}\text { le portiQue } & \text { Le Portique } \\ \text { Revue de philosophie et de sciences humaines }\end{array}$

$8 \mid 2001$

Nietzsche et le divin

\title{
Sans musique la vie serait une erreur
}

\section{Éric Blondel}

\section{OpenEdition}

\section{Journals}

\section{Édition électronique}

URL : http://journals.openedition.org/leportique/212

DOI : 10.4000/leportique.212

ISSN : $1777-5280$

\section{Éditeur}

Association "Les Amis du Portique"

Édition imprimée

Date de publication : 1 septembre 2001

ISSN : 1283-8594

\section{Référence électronique}

Éric Blondel, « Sans musique la vie serait une erreur », Le Portique [En ligne], 8 | 2001, mis en ligne le 09 mars 2005, consulté le 12 avril 2021. URL : http://journals.openedition.org/leportique/212 ; DOI : https://doi.org/10.4000/leportique.212

Ce document a été généré automatiquement le 12 avril 2021.

Tous droits réservés 


\title{
Sans musique la vie serait une erreur
}

\author{
Éric Blondel
}

"Sans musique la vie serait une erreur »

Crépuscule des idoles, Maximes et pointes, § 33.

1 Écoutons la phrase de Nietzsche citée en titre et en épigraphe. Nietzsche, très content de sa formule, l'a essayé sur deux correspondants (Peter Gast, compositeur et Georg Brandes, philosophe danois) avant d'en faire une maxime d'un de ses derniers ouvrages, Crépuscule des idoles, 1888. Il serait insuffisant, erroné, de ne voir dans cette magnifique déclaration d'amour à la musique, qu'un mouvement de passion personnelle. Nietzsche ne se contente pas de dire que lui, Frédéric Nietzsche, aime la musique à la folie : la chose serait émouvante, quoique relativement banale, même si elle est rare chez la gent philosophique. L'apophtegme ou, si vous y tenez, l'aphorisme, va bien au-delà. Très consciemment, Nietzsche, qui n'est pourtant plus guère coutumier $\mathrm{du}$ fait à la fin de sa carrière de philosophe, lui confère une portée proprement métaphysique. Il faut oser ce mot, souvent vilipendé par Nietzsche à cette époque, mais dont il faisait grand cas et usage intense lors de ses débuts philosophiques, en particulier dans la Naissance de la tragédie (1872). Dire que « sans musique, la vie serait une erreur ", c'est se placer au niveau des intentions du créateur, spéculer sur les desseins de Dieu : celui-ci aurait raté son œuvre, la Providence aurait manqué son but, la vie voulue par Dieu n'aurait pas de sens s'il y manquait la musique, tel est le sousentendu plaisant de Nietzsche qui, la connaissant fort bien, aurait pu plagier ainsi la formule de Voltaire (et le fait ici à sa manière) : si la musique n'existait pas, il faudrait l'inventer.

2 Mais chez Nietzsche, il n'y a pas de Dieu, pas de théodicée. Et cette formule métaphysique est ici forgée par lui à usage parodique ou, si l'on peut dire, «rétro »: mais, paradoxalement, elle n'en acquiert que plus de valeur. Si Dieu est mort, la Vie est la seule réalité : et si cette réalité doit être inconditionnellement affirmée, si la pas vie n'a pas à être niée ou discréditée, elle doit être la réalité absolue, celle qu'on ne doit pas simplement subir, supporter, dissimuler ni travestir. Donc : la musique ne constitue pas 
un des agréments accessoires, utiles ou nécessaires de la vie, elle est, bien plus, le signe de la perfection de la vie, elle exprime la vie en soi, en tant que telle, dans sa perfection, dans son essence la plus intime. Telle est la conviction de Nietzsche, inébranlable, d'un bout à l'autre de sa fluctuante carrière, qu'il s'exprime dans ou hors de la métaphysique, et en dépit de toutes les vicissitudes de sa pensée changeante et en devenir. Sans musique, la vie serait une erreur, de même que, sans pardon, bonté ou infinitude, Dieu serait une erreur, un concept raté ou un diable boiteux. La musique est partie intégrante de l'essence de la vie ou, si, comme Nietzsche, on se méfie de ce mot emprisonnant, de sa " perfection ».

3 Mais quelle musique, et en quel sens la musique définit-elle la vie et exprime-t-elle le fond et la perfection de la vie? Les préférences et les dégoûts de Nietzsche en matière de musique (genres, styles, compositeurs, techniques et contrapuntiques) vont de pair avec sa psychologie et son histoire personnelle, mais ils sont également fonction, plus largement, de sa représentation du monde, de ses choix idéologiques et philosophiques. Et l'on ne saurait oublier, à cet égard, que la musique est tellement liée à tous les aspects de la vie de Nietzsche qu'il a non seulement beaucoup écrit sur la musique de son temps (Wagner...) et la musique en général mais encore tâté lui-même de la composition.

4 Demandons-nous donc plutôt quelle musique écoutait Nietzsche, à laquelle allait sa prédilection, ce qui pourra nous conduire vers le problème philosophique essentiel pour Nietzsche, de savoir quel rôle peut jouer et quel rôle on peut assigner à la musique dans la civilisation et la culture. La question des goûts de Nietzsche est difficile, car il brouille psychologiquement les cartes par l'exercice impitoyable de l'esprit critique moral et philosophique contre ses amours les plus passionnées. Chez lui, c'est encore l'admiration et 1'amour qui s'expriment dans les méchancetés polémiques. Cela vaut pour les grands philosophes, pour le christianisme et l'exigence morale. Mais c'est encore plus vrai pour la musique, et pour la musique allemande en particulier. Par exemple, il ne faut pas se méprendre sur l'opinion de Nietzsche à l'égard de la musique de Wagner, il ne l'a pas aimée à la folie pour ensuite la détester et la critiquer, il n'a pas fini par brûler ce qu'il avait commencé par adorer, bien au contraire, il ne s'est détaché par la critique que de ce qu'il a aimé, il a attaqué dans Wagner son propre penchant excessif pour cette musique, son propre wagnérisme, son propre romantisme, sa propre décadence. Pour qui lui est indifférent, en musique ou en littérature, Nietzsche n'a que le silence, tandis que sa façon d'aimer doit passer par le blasphème, l'attaque brutale, que l'on doit percevoir et comprendre comme la mise en cause des faibles, ou de la faiblesse de Nietzsche lui-même et des mélomanes «hypocrites, ses semblables, ses frères ». Et vaut pour lui, par excellence, ce qu'il écrit dans le Cas Wagner : « on est attiré par ce qu'on devrait repousser ${ }^{1}$.

Qui Nietzsche a-t-il repoussé de tous les musiciens qu'il admirait et qui l'attiraient dans une certaine mesure (avouée) malgré lui ? Wagner, bien sûr, mais il est un symbole, un symptôme, un condensé de la musique allemande, de la civilisation allemande, du romantisme allemand, du christianisme larvé dans la morale et dans l'art de tout ce que Nietzsche aimait d'un amour déçu et vengeur, de «l'amour qu'on a pour une femme dont on doute ${ }^{2}$, ce qui est vrai autant de Socrate que de la vie et de sainte Cécile (fragment de 1871 sur musique et langage) ${ }^{3}$. L'attitude ambivalente de Nietzsche envers la musique de Wagner est révélatrice de ce qu'on peut attendre et de ce qu'on 
doit refuser de la musique en général. Qu'est-ce donc que Wagner et la musique ont donné à Nietzsche et quelle attente ont-ils déçue et trahie ?

C'est à la fois psychologique, physiologique, moral et métaphysique, et on peut résumer et symboliser tout cet ensemble par la doctrine et sous le nom de Schopenhauer, le maitre à penser de Nietzsche. Dans le chapitre du Monde comme volonté et comme représentation, la seconde «Bible » de Nietzsche, Schopenhauer écrit que la musique est expression du monde, plus encore, de l'être vrai des choses, de la réalité intime, en soi du monde, c'est-à-dire de la volonté. Coïncidant avec le monde, la musique « est une copie aussi immédiate de toute la volonté que l'est le monde ${ }^{4} »$. Nietzsche, qui cite plusieurs pages de ce texte de Schopenhauer dans le paragraphe 16 de la Naissance de la tragédie, résume ainsi la teneur de ces lignes : «Selon Schopenhauer, nous comprenons donc la musique immédiatement comme langage de la volonté ${ }^{5} "$. Mais, comme la volonté est la réalité intime des choses, la chose en soi, l'essence cachée et inconsciente des phénomènes, la musique est le vrai langage de la réalité, elle s'identifie avec la réalité en soi, elle est une expression métaphysique et directe, plus vraie que les mots du langage, du monde comme volonté. Elle a donc valeur métaphysique, d'expression de la volonté comme réalité en soi et profonde des phénomènes, en deçà des autres formes superficielles de représentations, par exemple la littérature. Et c'est en ce sens d'abord que Nietzsche, dans la Naissance de la tragédie, l'appelle l'art dionysiaque, comme le drame musical grec, par opposition aux arts de la représentation, aux arts plastiques de la forme extérieure phénoménale, les arts appoliniens. "Ce qui distingue la musique des autres choses, écrivait Schopenhauer cité et approuvé par Nietzsche, c'est qu'elle n'est pas une reproduction du phénomène ou, pour mieux dire, de l'objectivité adéquate de la volonté et que par conséquent elle présente a tout ce qu'il y a de physique dans le monde, le métaphysique, à l'ensemble des phénomènes, la chose en soi ». Et Schopenhauer précisait: «Le compositeur nous révèle l'essence intime du monde, il se fait l'interprète de la sagesse la plus profonde, et dans une langue que sa raison ne comprend pas, de même la somnambule dévoile, sous l'influence du magnétiseur, des choses dont elle n'a aucune notion, lorsqu'elle est éveillée ${ }^{6}{ }^{\prime}$. Ainsi, et Nietzsche reprend cette théorie tout comme Wagner (afin de la lui jeter plus tard à la tête !) : « La musique est un exercice de métaphysique inconscient dans lequel l'esprit ne sait pas qu'il fait de la philosophie ${ }^{7} »$.

7 Mais qu'est-ce que cela veut dire, concrètement? D'abord, au plan proprement métaphysique, que la musique est la révélation du monde, une vérité plus profonde que toute religion et toute philosophie, comme déjà l'écrivait Beethoven à peu près au même moment que Schopenhauer. La musique dit le monde, dit ce que les mots ne peuvent exprimer, elle révèle la réalité plus profondément que tout autre mode d'expression et même que tout autre art. « C'est un art si élevé, écrivait Schopenhauer, et si admirable, si propre à émouvoir nos sentiments les plus intimes, si profondément et si entièrement compris, semblable à une langue universelle qui ne le cède pas en clarté à l'intuition elle-même ${ }^{8}$ ». Mais la volonté, qu'est-ce d'autre que les désirs, les sentiments, les affects divers, la joie et les souffrances des hommes en particulier? Dans le Drame musical grec, Nietzsche cite à nouveau Schopenhauer pour le reprendre à son compte: "La musique touche immédiatement le cœur, car elle est la véritable langue universelle, partout comprise ${ }^{9} »$. Du point de vue psychologique, Nietzsche reprendra cette idée en écrivant dans Par-delà le bien et le mal (\$ 106) que, "grâce à la 
musique, les passions jouissent d'elles-mêmes ». Ou encore : « La musique [est] le reflet de toutes les activités et conduites humaines » (Le Voyageur... § 156).

8 C'est là le grand point, pour Nietzsche: même abandonnée la théorie schopenhauérienne de la volonté, comme trop métaphysique, il demeure que la vie est affects, passions, c'est-à-dire volonté de puissance. Et alors toute la conception nietzschéenne de la musique devient claire : la musique exprime, mieux que tout autre art, la volonté de puissance, elle-même encore mieux traduite par l'art, «stimulant à la vie ", que par les autres véhicules et moyens d'expression, comme, par exemple, le langage : "menteur, sourd-muet et métaphysique ${ }^{10}$ ». Et, bien sûr, la musique peut traduire la volonté faible, la décadence, la négation de la vie, le renoncement religieux et moral à la vie, comme elle peut être aussi l'expression du vouloir affirmateur, du dire-oui à la vie, de la volonté de puissance forte, ascendante et créatrice.

Or c'est là que Nietzsche attendait beaucoup de la musique et qu'il s'estime trahi dans ses espérances. Wagner paie pour beaucoup d'autres, morts ou moins célèbres à l'époque, et le Cas Wagner ainsi que Nietzsche contre Wagner se résument à dire que "L'art de Wagner est malade " (Le Cas Wagner, §5), ce qui signifie que cette musique exprime une volonté de puissance, faible, décadente, des valeurs de négation de la vie. On n'a pas ici l'ambition de résumer ces ouvrages, alertes, durs, drôles, profonds et polémiquement superficiels. On se bornera à souligner les enjeux non seulement psychologiques, mais moraux et philosophiques qui s'attachent au rôle de la volonté décadente-faible dans la conception nietzschéenne de la musique, art par excellence dans une philosophie qui donne le privilège à l'art par rapport même à la philosophie, parce qu'il conduit ou, pour mieux dire, séduit en faveur de la vie (führt, verführt).

C'est en fonction de ce critère : les passions tristes ou la belle humeur (Heiterkeit), la négation ou l'affirmation de la vie, la force ou la faiblesse que Nietzsche évalue la musique, ce qui définit sa méthode généalogique : « En ce qui concerne toutes les valeurs esthétiques, je me sers désormais de cette distinction fondamentale : je me demande dans chaque cas particulier: "Est-ce ici la faim ou le surplus qui est devenu créateur?" "11". Prenant appui sur ce principe, extrait d'un texte fondamental qu'il faudrait relire en entier dans le détail, voici quelques exemples portant sur des compositeurs et des musiques. On en trouvera tout une anthologie dans les paragraphes 149 à 169 de la dernière partie d'Humain, trop humain, intitulée le Voyageur et son ombre, ainsi (\$161) sur Schumann «Le "jeune homme" tel que l'ont rêvé les romantiques auteurs de Lieder d'Allemagne et de France vers le premier tiers de ce siècle, ce jeune homme a été entièrement traduit en sons et chansons par Schumann, l'éternel jeune homme tout le temps qu'il se sentit en pleine possession de sa force ; il y a, il est vrai, des moments où sa musique rappelle l'éternelle "vieille fille" ». Ne nous hâtons pas trop de voir là du bel esprit facile ou du dédain. On a beau adorer Schumann, comme l'auteur de ces lignes (car Nietzsche en jouait et le connaissait assez bien pour ne pas aimer certaines de ses œuvres chorales), il faut reconnaître qu'il se définit par un romantisme énergique assez juvénile, avec notamment les Davidsbündlertänze ou les Kreisleriana. Nietzsche ignore ou passe sous silence des œuvres plus mystérieuses, rêveuses, mélancoliques ou shakespeariennes (telles que les Romances ou les Chants de l'aube, ou encore les Märchenbilder pour alto et piano). Il doit songer surtout à la Dichterliebe, d'après Heine, et aux Scènes d'enfants. Mais n'y a-t-il pas là, comme le susurre Nietzsche sans dire le mot (il est vrai inconnu à son époque !), une vision un peu Kitsch de l'énergie et de la jeunesse, une nuance de pureté et d'innocence 
moralisante, toute prête à s'étaler, à la faveur de circonstances telles que... la façade de vertu, d'amour de la nature et de la jeunesse du nazisme? Schumann n'y était pour rien, et il couvait aussi autre chose : mais il est indéniable qu'il a flatté en son temps certaines tendances de l'âme romantique pré-nazie que Nietzsche entravait dont il se méfiait. Comme l'a dit très joliment Thomas Mann, wagnérien et nietzschéen de bon aloi (au demeurant comme eux fort schopenhauérien), le précurseur, mais l'annonciateur, le prophète prêchant dans le désert du "pays plat allemand", la Cassandre du futur nazisme, dernier avatar criminel de certaines tendances humaines et allemandes du romantisme, dernière chance des faux durs, des jeunes « sains » et des " vieilles filles ». Et a-t-on su que Schumann avait été, comme ce fut le cas pour d'autres compositeurs allemands ou autrichiens, interdit par les nazis? C'est au moins parce que, comme dans Wagner, Beethoven ou Brahms, ils pouvaient reconnaître, à tort et à raison, des affinités avec leurs tendances.

11 Toutes ces remarques grossissent les indications de Nietzsche pour en montrer plus clairement le but subtil. Prendre Schumann pour un pré-nazi, direz-vous, quelle sottise ! C'est évident. Mais Nietzsche est un philosophe du soupçon, prompt à déceler les arrière-plans et arrière-pensées d'une musique, qui parfois trahissent une volonté "faible ", " décadente ". Ce que Nietzsche déclame à propos de Wagner n'est que le grossissement énorme, à la proportion de l'énormité du drame wagnérien, des petites remarques éparses du Voyageur et son ombre sur tel ou tel musicien allemand. Wagner, lui, a symbolisé ce que seuls de très rares courageux et lucides esprits comme Nietzsche attaquaient comme suspect, décadent, morbide, dangereux (" Wagner comme danger ») et qu'on peut en effet a posteriori considérer comme les prodromes de la maladie qui servait de principe inspirateur à l'Allemagne nazie (et, le sachant, Wagner, comme ses épigones: Strauss, Mengelberg et Karajan, aurait laissé faire ou pris position favorable) : pour Nietzsche, c'était certain dès le départ et il a usé sa voix et sa plume à crier pour attirer l'attention sur les tendances malsaines de la germanité que, selon lui, Wagner résumait comme un symptôme à son époque. La sœur de Nietzsche, dans l'infaillibilité de sa sottise, ne s'y est pas trompée : elle a trahi son frère au profit de ses plus mortels ennemis idéologiques et musicaux, le pangermanisme, le romantisme, l'historicisme et le théâtralisme (antisémite) de Wagner - et la négation du vouloirvivre, la musique comme négation de la vie en Parsifal, «pur innocent» comme dit Nietzsche, le parfait nigaud (Reine Tor).

12 Mais si c'est la musique que refuse Nietzsche, que veut-il ? Ne lui parlez pas de Bizet : il n'a servi qu'à faire pièce à Wagner, que Nietzsche, dans sa méfiance même, place incommensurablement plus haut. Ce que Nietzsche veut de la musique, c'est la «belle humeur» contre la «mélancolie de l'impuissance». Mozart contre ce qu'il croit pouvoir entendre dans Brahms. Ou encore : la force, la puissance tectonique, contre la «mélodie infinie»-Beethoven contre Wagner. Ou bien encore : la création contre la faiblesse épigonale. Bach ou Haendel contre - encore une fois - Brahms (mais au fond Wagner). «À entendre la musique de Bach, nous aurons l'impression (pour le dire à la manière élevée de Goethe) d'être présents au moment même où Dieu créa la monde » ${ }^{12}$. De même pour Haendel : « Hardiment novateur dans l'invention de sa musique, véridique, puissant, tourné par affinité vers l'héroïsme dont un peuple est capable... » ${ }^{13}$.

13 Mais Mozart! Mais la «belle humeur ", la Heiterkeit! Ces mots résument tout ce que Nietzsche attend de la musique, et dont voici quelques échantillons : "Beethoven et Mozart. La musique de Beethoven [...]. Innocence de la mélodie : c'est de la musique sur de 
la musique. [...] Ses "mélodies" [...] lui sont des réminiscences transfigurées d'un "monde meilleur", un peu comme Platon imaginait ses Idées. - Mozart a de tout autres rapports avec ses mélodies; il ne trouve pas ses inspirations en écoutant de la musique, mais en regardant la vie, la vie la plus animée » ${ }^{14}$. Deux aphorismes plus loin, Nietzsche explique ce qu'il entend par cette "heitere Musik», cette "musique gaie " [l'adjectif qualificatif étant mis car l'auteur entre guillemets pour en signaler le sens subtil]. Le mot heiter désigne ce qui est à la fois serein malgré le malheur ou la blessure, et gai par une certaine force de résistance à la tristesse et au désespoir allégrement surmontés. C'est, comme l'expliquera plus tard la préface au Crépuscule des idoles, une force d'âme qui conquiert la belle humeur: "Conserver sa belle humeur quand on s'est engagé dans une affaire et extrêmement exigeante, ce n'est pas une mince affaire : pourtant, quoi de plus indispensable que la belle humeur? Rien ne réussit lorsque fait défaut l'exubérance. Ce qui prouve la force, c'est le trop-plein de force [...]. La blessure même peut encore donner la force de se guérir ». Voici donc ce qu'écrit Nietzsche sur la "Musique de belle humeur »: "Quand on en a été longtemps privé, la musique passe ensuite trop vite dans le sang, comme un gros vin du Sud, et laisse l'âme engourdie comme par un narcotique, somnolente, toute à l'envie de dormir : c'est précisément ce que fait surtout la musique de belle humeur, qui donne à la fois l'amertume et la blessure, le dégoût et la nostalgie, et oblige à savourer et à resavourer tout cela comme dans un philtre empoisonné, mais sucré ${ }^{15}$ ». Cet effet secondaire, par contre-coup, c'est ce que Nietzsche veut, dans ses définitions ultérieures de la musique, éviter: qu'elle soit mélancolique ou gaie, profonde ou légère, désinvolte on nostalgique, la musique doit tonifier l'âme, produire des sentiments de force et l'activité vitale dans l'esprit et dans le corps, rendre actif (comme eût dit Spinoza) et créateur (comme le répète sans cesse Nietzsche) : «Mon pied a besoin de cadence, de danse, de marche (or, au son du "Kaisermarsch" de Wagner, même le jeune Kaiser serait bien en peine de marcher au pas !) - c'est qu'il exige avant tout de la musique l'ivresse de bien marcher, de bien aller au pas, de bien danser. Mais mon estomac ne proteste-t-il pas lui aussi ? Et mon cœur ? Ma circulation sanguine? Mes entrailles ne s'affligent-elles pas? Est-ce que, sans y prendre garde, je ne m'enroue pas ?... Pour écouter Wagner, j'ai besoin de pastilles Géraudel... Cela m'amène à poser la question suivante : qu'attends donc de la musique mon corps tout entier ? Car l'âme, cela n'existe pas... Je pense que c'est de s'alléger. C'est comme si toutes les fonctions animales avaient besoin d'être stimulées par des rythmes légers, pleins d'allant, assurés ; comme si l'or des mélodies tendres, onctueuses, libérait de sa pesanteur la vie d'airain et de plomb. Ma mélancolie entend trouver le repos dans les réduits et les abîmes de la perfection: c'est pour cela que j'ai besoin de la musique. Mais Wagner rend malade - que m'importe à moi le théâtre? Que m'importent les transes de ses extases "morales" 16 ".

Faute de pouvoir commenter ce texte comme il le mériterait, lento et appassionato, on se contentera de deux remarques essentielles: d'abord, la musique est placée par Nietzsche sur le plan des affects, de la volonté et du corps, ce qui explique que Nietzsche exclue l'âme, comme siège des pensées morales ou transcendantes et abstraites ou idéales ; et ensuite, le but qu'il lui assigne est, non pas la gaieté à tout prix, dont on a vu plus haut les effets d'après-coup, comme le mal aux cheveux après l'ivresse, mais la perfection, l'achèvement des états du corps et du désir, surtout des affects, sentiments et passions, y compris par le jeu cathartique et reposant (ou apaisant) de la mélancolie. Les passions doivent ainsi se "spiritualiser" ou se «sublimer » par les rythmes, les mélodies et les harmonies de la musique. C'est ainsi 
que le corps et l'âme deviennent légers, « de belle humeur » : autrement dit, la musique est non point un narcotique, un opium du peuple ("et qui n'est pas "peuple", en l'occurrence?" demande Nietzsche dans la suite du texte), mais le moyen de la Selbstüberwindung, du dépassement de soi, de l'accomplissement physique et moral sans négation de soi et sans négation de la vie ni du corps. Cette paix de l'accomplissement, Nietzsche l'appelle parfois aussi le bonheur et, souvent se sert du terme d'" alcyonien " pour en définir la nature et se situer lui-même affectivement et esthétiquement. La mythologie nous dit que les alcyons sont des oiseaux qui annoncent par leur venue le calme de la mer agitée : c'est ainsi que les voyait aussi Chénier dans la Belle Tarentine. Cela fait songer Nietzsche au Midi, à l'Italie, à la Grèce, à Lucrèce et à Épicure, à Claude Le Lorrain et à Poussin, surtout à l'époque d'Humain, trop humain et du Gai Savoir ${ }^{17}$. C'est pourquoi Nietzsche dans le même texte d'Ecce Homo ${ }^{18}$, fait une équivalence entre le bonheur et le Midi et dit préférer «tout ce qui a poussé de l'autre côté des Alpes, je veux dire de ce côté-ci ${ }^{19}$ et fait une fleur à Rossini et à son " maestro vénitien Pietro Gasti » [Peter Gast], « mon Midi à moi ». Et, une décennie plus tard, il retrouve dans ce texte de 1888, les mêmes mots pour caractériser ce bonheur et le symbolise identiquement sous le nom, inattendu, de Chopin, comme dans la paragraphe $160 \mathrm{du}$ Voyageur et son ombre: «La Barcarolle de Chopin. Presque toutes les situations et manières de vivre ont leur moment de bonheur. C'est lui que les bons artistes savent prendre au filet. Même l'existence au bord de l'eau a le sien, elle si ennuyeuse, si sale, si malsaine à vivre et côtoyant la canaille la plus bruyante et la plus cupide - ce moment de bonheur, Chopin l'a si bien fait chanter, dans la Barcarolle, qu'à l'écouter l'envie pourrait prendre même les dieux de passer de longues soirées d'été allongés dans une barque " ${ }^{20}$. Et dans Ecce Homo et Nietzsche contre Wagner: «Je n'ajouterai qu'un mot, à l'intention des oreilles élues : ce que, quant à moi, j'attends exactement de la musique. Qu'elle soit gaie [de belle humeur: heiter] et profonde, comme un après-midi d'octobre. Qu'elle soit personnelle, folâtre, tendre [difficile, désinvolte, tendre : c'est le portrait de Carmen qui commence à s'esquisser], une douce petite femme pleine de malice et de grâce [d'abjection et de grâce]... Je n'admettrai jamais qu'un Allemand puisse seulement savoir ce qu'est la musique. Ce que l'on nomme les musiciens allemands, à commencer par les plus grands, sont tous des étrangers, Slaves, Croates, Italiens, Néerlandais ou juifs [on aurait aimé que la sœur de Nietzsche et les nazis n'oublient pas ce passage dans leurs évocations pseudos-nietzschéennes]... ou, si ce n'est le cas, ce sont des Allemands de la forte race allemande maintenant éteinte, tels que Heinrich Schütz, Bach et Haendel. [Idem: le prétendu précurseur du nazisme évoque souvent Beethoven, d'origine flamande, Mendelssohn, juif, et se sent soulagé que Mozart n'ait pas été Allemand, mais Autrichien !] Quant à moi [écoutons bien !], je suis encore assez Polonais pour cela, je donnerais pour Chopin tout le reste de la musique: j'en excepte, pour trois raisons différentes, Siegfried idyll de Wagner, peut-être Liszt qui, par ses nobles accents orchestraux, l'emporte sur tous les autres; enfin tout ce qui a poussé de l'autre côté des Alpes, je veux dire de ce côté-ci. [...] Quand je cherche un synonyme à "musique", je ne trouve jamais que le nom de Venise. Je ne fais pas de différence entre la musique et les larmes - je ne peux imaginer le bonheur, le Midi, sans un frisson d'appréhension » (Nietzsche contre Wagner).

15 Tout le reste, pourquoi Nietzsche veut-il l'ignorer ou le rejeter, comme allemand, morbide, décadent, etc. ? Seulement parce que, dans sa façon de traiter les affects, de négliger la construction, la force, la ligne mélodique, la musique, chaque fois, pousse l'auditeur à nier la vie, se réfugier dans la négation, la calomnie de la vie et du corps, 
dans ce que Nietzsche, le reprochant injustement à Brahms pour en accabler en fait Wagner par ricochet, appelle " la mélancolie de l'impuissance » (le Cas Wagner, second Post-Scriptum) : "Cinquante pas plus loin, écrit-il, on trouve la wagnérienne », et il définit ainsi l'effet de cette musique d'imposteur, enchanteur, ensorceleur et histrion hypnotiseur: "Come si dorme con questa musica! ${ }^{21}$ ». Alors, et dans ce cas seulement, décrite et diagnostiquée tout au long dans le Cas Wagner, la musique est considérée par Nietzsche d'une oreille soupçonneuse, comme Circé, comme "venant des Sirène", comme ce qui dirige vers la mort et « séduit ou induit en la tentation» de la perdition. Et son attitude en ce cas est ambiguë : "C'est une vielle superstition des philosophes que de croire que toute musique provient des Sirènes ${ }^{22}$. Peut-être, écrit-il dans ses notes posthumes, la musique est-elle l'art par définition qui naît en des époques de faiblesse, de décadence, d'abandon à la mort. Peut-être toute musique est-elle «sans avenir » : «C'est dans la musique de Beethoven et de Rossini qu'expire mélodieusement le XVIII ${ }^{\mathrm{e}}$ siècle, le siècle de la rêverie, de l'idéal brisé, du fugace bonheur. Toute musique vraie, toute musique originale, est un chant de cygne. - Peut-être notre musique moderne, quel que soit son empire - et sa tyrannie, n'a-t-elle plus devant elle qu'un court laps de temps; car elle a surgi d'une culture dont le sol miné s'enfonce rapidement, d'une culture bientôt engloutie ${ }^{23}$.

La grande musique, comme la "morale des seigneurs ", " dit oui », « elle dispense un peu de sa richesse aux choses - elle transfigure le monde, l'embellit, lui donne du sens ${ }^{24}$. La musique décadente, comme celle de Wagner, "défend en secret ce qui présente un danger mortel et dénigre ce monde ", "flatte tous les instincts nihilistes (bouddhistes) et les travestit en musique », «brise l'esprit ». "La musique, nouvelle Circé... Sa dernière œuvre est, sous ce rapport, son plus grand chef-d'œuvre. Parsifal... ${ }^{25}$ ».

17 Toujours dans Nietzsche contre Wagner, qui est un collage de textes antérieurs retravaillés et à peine modifiés, Nietzsche reprend un paragraphe $(\$ 165)$ du Voyageur et son ombre, vieux de dix ans, sans importantes modifications. «Faire parler un langage dramatique à la musique cela n'est-il pas proprement un péché contre l'esprit de Mozart, esprit de belle humeur (heiter), enthousiaste, tendre, amoureux, qui par bonheur n'était pas Allemand [ajout de 1888 !] et dont le sérieux était un sérieux plein d'or et de bonté, et justement pas le sérieux d'un bon bourgeois allemand... Pour ne rien dire du sérieux du "convive de pierre"... Mais vous êtes tous persuadés que toute musique est musique du "convive de pierre" " (Wagner comme danger).

On comprend mieux, musicalement pour ainsi dire, les raisons qui, dans une lettre à Gast du 15 juin 1888, ont poussé Nietzsche à ajouter quelques mots de précision à la formule qui lui sert dans le Crépuscule des idoles et qui nous a servi d'épigraphe solennelle et de titre. Cette fois, Nietzsche ajoute deux mots : « La vie sans musique est tout simplement une erreur, une torture, un exil». Seul l'amour déserté ou la crainte que la solitude désertique ne croisse ou n'advienne peut faire proférer ces si lamentables plaintes. Une erreur, une torture, un exil : c'est si la vie vous abandonne, si la maladie menace, la décadence, la faiblesse, le désespoir, le ressentiment, c'est si « le désert croit ", lieu de mort, de solitude et de silence. La musique, alors, est la vie, la vie ascendante et créatrice, qui sait s'illuminer contre la torture et l'exil.

Et, comme dirait le frère de Nietzsche en angoisse, en solitude, en torture et en grimaces bouffonnes de martyr aux mille masques : «The rest is silence ». 


\section{NOTES}

1.. Le Cas Wagner, $\S 5$.

2.. Le Gai Savoir, préface.

3.. Et encore une fois, la citation ou paraphrase de Schopenhauer (fin du chapitre du Monde... mentionné ci-après), qui évoque la Sainte Cécile de Raphaël, évocation reprise sans façons par Nietzsche dans Richard Wagner à Bayreuth (quatrième Considération intempestive), § 9.

4.. SCHOPENHAUER, Le Monde comme volonté et comme représentation, trad. Burdeau revue et corrigée par R. Roos, Paris, 1966, p. 329.

5.. NIETZSCHE, La Naissance de la tragédie, trad. Lacoue-Labarthe, Paris, 1972, § 16, p. 113.

6.. SCHOPENHAUER, loc. cit., p. 332-333.

7.. Ibid., p. 338.

8.. SCHOPENHAUER, ibid., p. 327.

9.. « Le drame musical grec ", Écrits posthumes, 1870-1873, trad. Backès, Paris, 1975. Nietzsche cite Schopenhauer sans guillemets.

10.. Crépuscule des idoles, Raids d'un intempestifs, § 26.

11.. Le Gai Savoir, § 370.

12.. Le Voyageur et son ombre, $§ 149$.

13.. Ibid., § 150.

14.. Le Voyageur et son ombre, § 152 .

15.. Humain, trop humain, II, Le Voyageur et son ombre, trad. Rovini (modifiée), Paris, $1968, \S 154$.

16.. Ecce Homo, trad. Hémery, Paris, 1974, II, § 7.

17.. Cf. le $\S 152$ du Voyageur et son ombre, et le $\S 295$, qui décrit un paysage idyllique.

18. Repris dans l'« Intermezzo » de Nietzsche contre Wagner.

19.. Nietzsche contre Wagner est sous-titré Lettre de Turin.

20.. Le Voyageur, loc. cit., trad. citée.

21.. Fragments posthumes 1887-1888, trad. Klossowski, Paris, 1976 : il s'agit du Prélude de Lohengrin!

22.. Le Gai Savoir, § 372.

23.. Nietzsche contre Wagner, « Une musique sans avenir », trad. citée. Encore une allusion à « la musique de l'avenir » de Wagner. Cf. aussi le $§ 3$ de la préface d'Humain, trop humain, II, « Cave musicam »!

24.. Le Cas Wagner, "Épilogue », trad. citée.

25.. Ibid., Post-Scriptum.

\section{RÉSUMÉS}

Nietzsche est sans doute le premier philosophe pour qui la musique revête une si grande importance. Certes, la métaphysique de Schopenhauer l'avait incité à soupçonner que cet art 
nous mettait en relation avec «l'essence intime du monde ». Mais une fois même abandonnée sa " métaphysique artiste », Nietzsche continue à accorder à la musique une importance insigne. On tente ici une interprétation de cette fidélité et de cet attachement. 